\title{
Conference Abstracts
}

\section{A Case of Medullary Carcinoma of the Duodenum Treated with Pembrolizumab}

\author{
Louisa Liu, MD1', Simmer Kaur, MD², Rahel Demisse, MD² \\ 1 University of California, Riverside School of Medicine, ${ }^{2}$ Loma Linda University Medical Center \\ Keywords: medullary carcinoma, pembrolizumab, colorectal cancer \\ https://doi.org/10.53876/001c.28392
}

\section{International Journal of Cancer Care and Delivery}

Vol. 1, Issue Supplement 1, 2021

\section{INTRODUCTION:}

Medullary carcinoma (MC) is a recognized histologic subtype of colorectal cancer characterized by poor glandular differentiation and intraepithelial lymphocytic infiltrate. However, MC originating from the small intestine is exceedingly rare, with only eight cases described in the literature. We report the first case of a patient who presented with unresectable MSI-H medullary carcinoma of the duodenum and was treated with pembrolizumab.

\section{CASE PRESENTATION:}

A 50-year-old man with history of adenocarcinoma of the transverse colon $\mathrm{s} / \mathrm{p}$ hemicolectomy and adjuvant treatment with chemoradiation in 2019 and family history of Lynch syndrome presented with abdominal pain for two weeks. CT abdomen/pelvis revealed a $10.7 \mathrm{~cm}$ by $4.3 \mathrm{~cm}$ mass in the mid-portion of the duodenum abutting against the pancreatic head. EGD demonstrated circumferential, partially obstructing, intrinsic stenosis of the duodenum with ampullary involvement and likely invasion into the pancreatic head and common bile duct. Endoscopic biopsy of the primary tumor revealed poorly differentiated medullary carcinoma. Immunohistochemical staining showed loss of MLH1 and PMS2 expression. Staging with CT chest showed no evidence of disease. PET scan redemonstrated circumferential duodenal wall thickening and hypermetabolic activity with SUV max of 26.4 , as well as PETavid epigastric, retroperitoneal, and periaortic lymphadenopathy suggestive of metastasis. He was recently started on pembrolizumab and remained in follow-up.

\section{DISCUSSION:}

Due to the rarity of the tumor, there is no standardized approach to treatment. All patients in previously published cases underwent surgical resection. However, our patient was deemed a poor surgical candidate. Given his previous history of colon cancer and treatment with platinum-based therapy, he qualified for pembrolizumab as first-line therapy for his MSI-H tumor. To our knowledge, this is the first report of MC of the duodenum and the first MC to be treated with pembrolizumab in the first-line setting. 